\title{
Género y currículum de formación del profesorado en Educación Física: un diálogo entre Brasil y España
}

\section{Gender and teacher training curriculum in Physical Education: a dia- logue between Brazil and Spain}

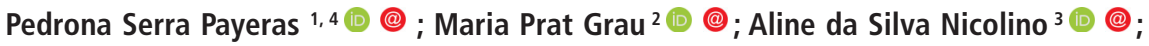 \\ Susanna Soler Prat ${ }^{1}$ (1) @; ; Ana Márcia Silva ${ }^{3}$ (1) @ \\ 1 Institut Nacional d'Educació Física de Catalunya (INEFC), Universitat de Barcelona (UB), \\ España; ${ }^{2}$ Universitat Autònoma de Barcelona (UAB), España; ${ }^{3}$ Universidade Federal de \\ Goiás (UFG), Brasil; ${ }^{4}$ Universitat de les Illes Balears (UIB), España.
}

\section{Resumen}

En este artículo se pretende analizar cómo se ha introducido la perspectiva de género en los grados universitarios que capacitan al profesorado de educación física en Brasil y España. La investigación se ha desarrollado a partir del análisis de contenido de los proyectos curriculares o guías docentes de 65 instituciones de educación superior brasileñas (7,8\% del total) y 16 universidades españolas (45\%) que estaban disponibles en línea. Para analizar y discutir los datos del artículo se ha utilizado la teoría iniciada por Tetreault (1985), en la que destaca cinco niveles de inclusión del conocimiento sobre las mujeres y las relaciones de género. La investigación ha permitido constatar que la presencia de la perspectiva de género es puntual y anecdótica en el proceso de formación de los y las profesionales de ambos países a pesar de la existencia de marcos legales que establecen la necesidad de incorporarla. Los resultados confirman que la construcción del conocimiento y las diversas formas de transmitirlo influyen en la transformación o la reproducción de las relaciones hegemónicas de género. Las aportaciones de este estudio muestran la necesidad de cuestionar la situación actual y apelar a la reflexión institucional en ambos contextos para incorporar cambios sustanciales en la formación del profesorado y así fomentar la transformación social desde las universidades.

Palabras clave: Formación del profesorado; Género; Educación Física; Educación Superior; Currículum.

\begin{abstract}
This article analyses the inclusion of the gender perspective in the training of physical education teachers in Brazil and Spain. The research has been developed from the analysis of the content of curricular projects or subject guides of 65 Brazilian higher education institutions (7.8\% of the total) and 16 Spanish universities (45\%) that were available online. The theory initiated by Tetreault (1985), which highlights five levels of inclusion of knowledge about women and gender relations, has been used to analyze and discuss the data in the article. The research has shown that the presence of gender is punctual and anecdotal in the training process of professionals from both countries despite the existence of legal frameworks that establish the need to incorporate it. The results show how the construction of knowledge, and the various ways in which it can be transmitted entails different implications for the greater or lesser transformation of gender relations. Contribution of this study show the need to question the current situation and appeal to institutional debate in both contexts in order to incorporate substantial changes in teacher training and thus foster social transformation from the universities.
\end{abstract}

Keywords: Teacher training; Gender; Physical Education; Higher Education; Curricula.

Recomendación de cómo citar este artículo (APA 6)

Serra, P., Prat, M., Nicolino, A., Soler, S. y Silva, A. M. (2020). Género y currículum de formación del profesorado en Educación Física: un diálogo entre Brasil y España. Revista Iberoamericana de Educación, 82(2),191-210. https://doi.org/10.35362/rie8223637 


\section{Género y educación física: reflexiones introductorias}

Entre los ocho objetivos del milenio de la Organización de las Naciones Unidas, se estableció el de la igualdad y el empoderamiento de la mujer. Sin embargo, el informe del 2015 refleja grandes diferencias en función de los países, situando Latinoamérica entre las zonas donde se dan disparidades más extremas (ONU, 2015). De acuerdo con datos del Atlas da Violência (2018), Brasil es el quinto país en número de feminicidios y el tercero en números absolutos, lo que representa un 40\% del total de feminicídios en América Latina y Caribe (ONU, 2019). Por ello, en la Agenda del 2030, entre los 17 objetivos planteados, continúa presente la necesidad de establecer políticas de igualdad entre hombres y mujeres en todos los niveles educativos (ONU, 2015), pues "la formación docente sigue incorporando poco las discusiones referentes al género y la sexualidad" (UNESCO, 2013, p.4).

Desde la década de 1990 en Europa y América Latina, un gran número de instituciones, organismos nacionales e internacionales, han articulado políticas sociales y educativas para que la temática de género y la igualdad de oportunidades se incorpore en la educación, como indican varios estudios (Blat Gimeno, 1994, Bonder, 1994). En este sentido, en los marcos legales y normativos de España y Brasil se observan varios cambios proactivos en esta cuestión.

En Brasil, a partir de los conocimientos de género y de las mobilizaciones sociales se experimentó una mayor liberdad de expresión y diversidad a raíz de la Lei de Diretrizes e Bases da Educação Nacional (LDB, no 9394/1996). En el 1997, se publicaron las directrices educativas a nivel nacional mediante los Parâmetros Curriculares Nacionais (Brasil, 1997). Con este documento, el primero publicado en Brasil, en el apartado de Pluralidade Cultural e Orientação Sexual, se incorporan las cuestiones de género y sexualidad, así como se dan las directrices para abordar la temática de forma transversal, en todas las áreas curriculares, incluïda la Educación Física. Sin embargo, desde el punto de vista formativo, no será hasta el año 2015 cuando el Consejo Nacioal de Educación (Conselho Nacional de Educação) concretará una diretriz curricular nacional para la formación docente (Resolução CNE N 02 de 2015), donde se contemplan las temáticas de género y sexualidad. No obstante, en el actual escenario político del país se observa un movimiento reaccionario que logra retirar del Plano Nacional de Educação (PNE 2014-2024) el énfasis existente hasta el momento centrado en la promoción de la igualdad racial, regional, de género y de orientación sexual (Rosado-Nunes, 2015). Más 
actualmente, se identifica la supresión de los conceptos de género, identidad de género y orientación sexual de documentos educacionales del gobierno federal (Antas y Arantes, 2019).

En el caso de España, en el marco del proceso de construcción del Espacio Europeo de Educación Superior (EEES), se constata un esfuerzo institucional para promover la igualdad de oportunidades en los ámbitos educativo y universitario. Un impulso importante lo desarrolla la Ley Orgánica n. 3/2007, que fomenta la igualdad efectiva de mujeres y hombres, donde se regula la incorporación de la perspectiva de género como política pública así como su obligatoria inclusión en todos los grados universitarios, entre otros aspectos.

En ambos contextos, el desafío que se presenta en la universidad no se reduce al incremento de mujeres en sus aulas, sino que significa que la educación superior debe considerar la justicia social, referida a nociones de igualdad de oportunidades y de derechos humanos, en todas sus dimensiones (Donoso-Vázquez, Montané y Pessoa, 2014). El concepto de calidad universitaria debe recuperar el valor social, público, y de compromiso con la comunidad, ya que podemos considerar que no hay calidad sin equidad y justicia social (Seibold, 2000).

Incorporar la perspectiva de género en la educación superior requiere un compromiso de todas las disciplinas académicas para "desarrollar una mirada crítica hacia las discriminaciones en el estudiantado universitario, extraer del alumnado la cosmovisión cultural de género-poder y adoptar una posición activa/participante con responsabilidad en los cambios sociales" (Donoso-Vázquez, Montané y Pessoa, 2014, p.162). Así pues, la incorporación de la perspectiva de género en la educación superior, se puede dar en distintos niveles y desde diferentes perspectivas.

Para analizar las diferentes formas de inclusión de los conocimientos sobre las mujeres y las relaciones de género, desde los años 80 se han desarrollado varias escalas de valoración. Tetreault (1985) desarrolló una escala de cinco niveles en la inclusión de la perspectiva de género en el conocimiento de áreas como historia, psicología, literatura o antropología. El trabajo originario y sucesivas aplicaciones (Maher y Tetreault, 2011; Serra et al. 2016a) define los niveles de inclusión de la perspectiva de género en: conocimiento masculino; conocimiento compensatorio; conocimiento bifocal; conocimiento feminista; y conocimiento multifocal o relacional. El primer nivel, es aquel en el que el conocimiento masculino es considerado como universal, con una perspectiva totalmente androcéntrica, en que aquello propio del varón se considera general y válido para el conjunto de la población. En el segundo 
nivel, se pretende compensar el olvido generado en el primer nivel, es decir, la falta de referencias a la mujer como parte existente en el mundo. Por ello, en este nivel ya se hace mención explícita a algunas mujeres, aunque el contexto en el que se mencionan reproduce la idea que son casos anecdóticos. De esta forma se sigue reforzando la idea de que los hombres, y todo aquello relacionado con ellos, son la norma, la representación y el paradigma de los seres humanos. El tercer nivel plantea el conocimiento de forma bifocal. Este nivel ya presenta el conocimiento del mundo en dos dimensiones y tiene en cuenta la situación de las mujeres, si bien se hace de forma dicotómica, destacando aquello femenino y aquello masculino. En este caso se realzan las diferencias entre hombres y mujeres como colectivos homogéneos, ignorando la heterogeneidad existente en cada colectivo. Esta tercera fase se centra en la opresión de la mujer. El conocimiento feminista se considera el cuarto nivel de la escala. En este nivel se situa a las mujeres y todo aquello que las rodea en el centro de atención y da relevancia a su actuación en la vida y en la construcción del conocimiento. Se destaca la necesidad de describir al colectivo femenino como diverso ya que otras categorías sociales (edad, orientación sexual, condición económica, etc.) entran en juego en la definición del conocimiento. En el último nivel, se observa el enfoque multifocal, en el que se suma al planteamiento del nivel anterior, la experiencia y necesidades de las múltiples masculinidades. Por ello, este último nivel es el más completo y aporta una visión conjunta de los conocimientos y saberes femeninos y masculinos, eliminando la visión jerarquizada y dicotómica detectada en niveles anteriores.

De forma similar a la escala de Tetreault (1985), McIntosh (1984) propone el análisis de conocimiento a partir de cinco fases interactivas, que aplicó en el área de conocimiento de historia. La autora de este último trabajo añade que los niveles no son estáticos, ni exclusivos, ya que en un mismo momento se pueden dar diferentes niveles y enfoques. Por ello, en un mismo documento o curso se pueden dar elementos reproductores junto con elementos transformadores. Todos estos trabajos, han sido aplicados en el análisis de los libros de texto de escolares, planes educativos de grado superior, medios de comunicación y artículos científicos (Chuppa-Cornell, 2005).

Con estos cinco niveles es posible detectar distintos enfoques sobre como se realiza su inclusión: de la visión más androcéntrica del conocimiento en los materiales curriculares, a la visión crítica y transformadora de las situaciones de discriminación y desigualdad, superando la visión dicotómica de los géneros. 
En este marco, tal y como apuntan algunos trabajos (Devís-Devís et al., 2001), es preciso estudiar la incorporación de las categorías sociales, entre las cuales se encuentra el género, en los materiales curriculares en su sentido amplio (recursos pedagógicos, planificación de contenidos y la propia evalución). En este sentido, Svendsen y Svendsen (2014), definen que el discurso utilizado en los materiales curriculares por parte del profesorado universitario es una herramienta clave para la construcción de conomiento del alumnado universitario.

Es, en esta dirección, que este estudio se propone realizar un análisis para valorar hasta qué punto y cómo se incorpora la perspectiva de género en la formación del profesorado de educación física de España y Brasil. Para ello, se plantean los siguientes objetivos específicos: a) analizar la presencia (o ausencia) de género en el currículo formativo de formación de profesorado de educación física; b) identificar similitudes y diferencias a partir de un diálogo entre ambas realidades en lo que se refiere a los planes de formación. Se trata de una investigación que busca favorecer el intercambio mutuo de realidades y experiencias, así como tener un mapeo de la situación actual, tal y como proponen diversas investigaciones comparativas (Hernández, J., Gómez, R. H., Cinta, D., Carralero, A., y Altuve, E., 2008; Pereira e Silva, I., Pereira da Silva, J. M., Grabowski, G., y Zucchetti, D. T., 2017).

\section{Métodos y materiales}

Para la realización de esta investigación se estableció como fuente de investigación la documentación en la que se define el proceso de formación y el currículum de cada institución de educación superior, valiéndose de los siguientes criterios: 1. Ser un documento oficial de las instituciones de educación superior que ofrecen formación docente en educación física, denominados "Guias docentes" (GD) en España y "Proyectos pedagógicos y curriculares" (PPC), en Brasil; 2. Estar disponible en línea para su consulta; y 3. Contemplar una muestra de todas las regiones de cada uno de los países investigados. Se trata, en definitiva, de documentos que podemos designar como político-pedagógicos para no perder de vista esta dimensión de la educación superior y, en especial, de la formación docente.

En Brasil se analizó una muestra de 65 centros, de prácticamente todas las provincias, respetando la proporcionalidad de las cinco regiones que componen el país, y que representan un 7,8\% de los 826 centros de educación superior (públicos y privados), que impartían el grado denominado Licenciatura en educación física 
en 2015. En cada centro, se analizó el correspondiente "Proyecto pedagógico y curricular" (PPC), de modo que en el conjunto de los 65 PPC analizados se recogen un total de 3.074 asignaturas.

Por otra parte, en España se analizaron los documentos de 16 universidades públicas del total de 37 instituciones que impartían el grado en España en 2013 (una muestra del $45 \%$ de los centros), que supone el análisis de 763 guías docentes (GD).

Ambos documentos, los PPC brasileños y las GD españolas, si bien los primeros son mucho más extensos, entre 22 y 402 páginas (122,98 páginas), permiten conocer los objetivos o competencias generales establecidas en cada institución, el plan de estudios con las correspondientes asignaturas en cada centro, así como también las competencias y los objetivos específicos, los contenidos, las actividades formativas y de evaluación, y la bibliografía de cada asignatura.

Para analizar la información recogida de forma sistemática, se ha utilizado la técnica de análisis de contenido, que se aplicó en dos fases. En un primer momento, se realizó un análisis descriptivo y cuantitativo de documentos recogidos en cada país. En estos documentos se buscó las siguientes palabras clave por su relación directa con el objeto de investigación: coeducación, discriminación, equidad, feminismo, género, hombre(s), igualdad, mixto/a, mujer(es), y sexo, de acuerdo con el idioma original del documento. En una segunda fase, y a partir de los resultados del análisis descriptivo, se realizó un análisis del discurso de aquellos documentos que incorporaban algún término vinculado a la perspectiva de género.

A partir de un proceso deductivo-inductivo, según la forma de abordar la perspectiva de género y teniendo en cuenta la propuesta de Serra (2016), se estableció la siguiente categorización:

1. Ausencia de los conocimientos sobre las mujeres y su experiencia, cuando no hay ninguna referencia al género;

2. Presencia superficial o "políticamente correcta", cuando sólo se introduce en el apartado de "competencias";

3. Reproduciendo a mujeres y a hombres, implican la inclusión de conocimientos relacionados con la mujer y al hombre, pero la forma de presentarse puede inducir a reproducir los modelos tradicionales de género;

4. Reconociendo lo femenino y las múltiples diferencias, esta categoría guarda relación con el reconocimiento de las aportaciones y las experiencias de las mujeres y da valor a la cultura deportiva femenina, puesto que da visibilidad 
a la diversidad existente entre las diferentes mujeres, así como entre los hombres; y,

5. Transformación de las relaciones de género, se utiliza un lenguaje visibilizador y se reconocen las diferencias, se muestra un compromiso explícito con la promoción de la igualdad efectiva entre hombres y mujeres, y una voluntad de mejorar y transformar la realidad de cada ámbito considerando las múltiples identidades existentes.

Para ello, se ha utilizado el programa de análisis cualitativo NVivo®, versión 12 , como herramienta de apoyo.

\section{Resultados y discusión}

A continuación se muestran los resultados divididos en dos apartados. El primero hace referencia a la mayor o menor presencia de los términos vinculados a la perspectiva de género en los documentos de ambos países. El segundo apartado presenta los resultados del análisis del discurso de aquellos documentos en que aparece alguno de los términos. Para ello, se han identificado los documentos con las letras B (para referirse a los documentos de Brasil) o $E$ (cuando se trata de documentos de España), seguidos por el número correspondiente al documento codificado.

\subsection{La (in)visibilidad de la perspectiva de género}

El análisis de la presencia de términos vinculados a la perspectiva de género en todos los documentos político-pedagógicos permitió identificar 146 asignaturas que de alguna forma presentaron la perspectiva de género en la denominación de la asignatura, o en sus contenidos, objetivos, y/o bibliografía. En Brasil se identificaron 92 asignaturas, que representan un 3\% del total analizado, y en España se identificaron 54, que representan un $7 \%$ del conjunto de guías docentes analizadas.

Los datos cuantitativos muestran una presencia muy reducida de la perspetiva de género en la formación en educación física en ambos países, y constatan que no se introduce de forma transversal tal y como se recomienda desde el marco legal. Donoso-Vázquez, Montané y Pessoa (2014) exponen que el ámbito universitario se caracteriza por la poca o nula conciencia y sensibilidad sobre el tema, es decir, por la "ceguera en materia de género" por parte del profesorado. O bien no considera relevante o significativo prestar atención a la perspectiva de género en su tarea 
docente, o bien adopta una postura neoliberal en la que considera la desigualdad de género como un aspecto superado (Scraton, 2013). Bajo el discurso neoliberal se considera que la igualdad ya se ha alcanzado y que las posibles diferencias existentes se pueden explicar por una cuestión de "elección personal", en la que "si quieres, puedes" (Pfister, 2010). Entonces, se da la creencia de que si ya se ha conseguido la igualdad, no hace falta introducirla en la formación del profesorado de educación física (Prat y Flintoff, 2012).

Si analizamos la presencia de cada una de las palabras, destaca la ausencia del término "feminismo" en ambos países, ya que está presente solo en un PPC brasileño y en una GD española, siendo la palabra menos mencionada. La omisión de este concepto constata lo que Weiner (2000) denomina el "miedo al feminismo". Un "miedo" existente en el ámbito de la formación del profesorado europeo que genera, por ejemplo, una tendencia a considerar más adecuado el término género que feminismo, o incluso a considerar la perspectiva de género un tema tabú, por lo que se le discrimina y excluye del programa educativo.

En el campo de la educación física, la actividad física y el deporte, varias investigaciones han revelado una falta de interés y compromiso en relación con la equidad y la justicia social en general, e incluso han detectado sentimientos de rechazo e incomodidad entre el profesorado en formación frente a estos contenidos (Flintoff, 1993; Prat y Flintoff, 2012).

Resulta también relevante el análisis de la presencia de la palabra "hombre(s)" y "mujer(es)" ya que el lenguaje contribuye a la construción del imaginario y el pensamiento (Lledó, 2011). En Brasil, la palabra "hombre" es la más citada de las que se han buscado ya que aparece en 53 PPC (el 81,5\%), siendo utilizada como sinónimo de "ser humano". En los documentos españoles, aún siendo menor su presencia, su utilización se realiza con el mismo sentido que en Brasil. Un ejemplo es el que, entre otros ejemplos, contempla en el currículo una asignatura titulada "Homem, cultura e sociedade" (B52). O también en esta dirección se puede encontrar el tema "O jogo e o homem" en los contenidos de la asignatura "Bases Teórico-metodológicos do jogo" (B56). En España, citas como "la adaptación del hombre al medio que le rodea, lo que significa buscar una mejor integración humana..." (E12) o bien "se identifica con la idea del hombre como sujeto activo" (E24) son ejemplos del uso del sustantivo "hombre" para referirse a "ser humano". Estas referencias son representativas de prácticamente todos los docu- 
mentos que emplean el término "hombre" en su sentido genérico de la especie humana, pero que denota una perspectiva androcéntrica y sexista del lenguaje, como ha explicitado Lledó (2011).

Por contraste, en los documentos brasileños la palabra "mujer" fue citada en apenas 24 de los documentos. Y cuando se incorpora, la mayoría de estas citaciones aparencen solo en el título de algunas referencias bibliográficas, indicando una clara desigualdad de estatus en el uso de los términos "mujer" y "hombre". Una excepción importante es de un PPC que utiliza ambos términos (mujer y hombre) em una misma frase: "construir projetos de formação da docência profissional, tão necessário à educação, pressupõe, portanto, formar homens e mulheres capazes de intervir na realidade" (B1). En los documentos españoles, el término "mujer" también aparece en varios casos en las referencias bibliográficas, si bien puede aparacer también en el descriptor de un tema de la asignatura: "consideraciones nutricionales especiales en la práctica deportiva: deportistas niños y adolescentes, la mujer deportista; el deportista vegano,..." (E295), como una categoria especial a tratar. En raras ocasiones, aparecen las dos palabras clave juntas "aspectos diferenciales en el entrenamiento de mujeres y hombres" (E719).

Sin duda, el uso androcéntrico del lenguaje contribuye a falsear la realidad, mientras que un uso de un lenguaje no sexista mediante formas genéricas o duales permitiría poner de manifiesto cuando las mujeres están presentes en las guías docentes y si los conocimientos que se transmiten son igualmente válidos para unos y otras, ya que el hecho de "no nombrar" implica que se ignore esa realidad (Lledó, 2011). Así se puede observar como las ideologías, que en algunos casos se encuentran difuminadas, acaban saliendo a la luz a través del lenguaje utilizado, y a través de la omisión de determinados conceptos o términos que harían presente al colectivo femenino. Así, de una forma más o menos consciente se refuerza la ausencia de la perspectiva de género en la formación del futuro profesorado.

\subsection{Las diferentes formas de inclusión de la perspectiva de género}

Tras la descripción del nivel de (in)visibilidad de la perspectiva de género, a continuación se presenta el análisis del enfoque con el que se ha abordado la perspectiva de género en los documentos. Es decir, se examinan los discursos que quedan plasmados en los documentos analizados. En la tabla 1 se puede observar el porcentaje de documentos que se han clasificado según la categorización establecida (Serra, 2016). 
Tabla 1. Asignaturas recogidas en cada una de las categorías

\begin{tabular}{lcrrc}
\hline \multicolumn{1}{c}{ Categoría de análisis } & \multicolumn{2}{c}{ España } & \multicolumn{2}{c}{ Brasil } \\
\hline & $n$ & $\%$ & $n$ & $\%$ \\
\hline $\begin{array}{l}\text { Ausencia de los conocimientos sobre las } \\
\text { mujeres y su experiencia }\end{array}$ & 569 & 74,6 & 2.982 & 97,1 \\
\hline $\begin{array}{l}\text { Presencia superficial o "políticamente correcta" } \\
\text { Reproduciendo a mujeres (débiles, pasivas y }\end{array}$ & 141 & 18,4 & 64 & 2,1 \\
$\begin{array}{l}\text { oprimidas), y a hombres } \\
\text { Reconociendo lo femenino y las múltiples }\end{array}$ & 27 & 3,5 & 6 & 0,1 \\
diferencias & & 2,6 & 13 & 0,4 \\
\hline Transformación de las relaciones de género & 7 & 0,9 & 9 & 0,2 \\
\hline TOTAL & 763 & 100 & 3.074 & 100 \\
\hline
\end{tabular}

Fuente: Elaboración propia.

Las características de las asignaturas que se encuentran en cada categoría, desde un punto de vista cualitativo, y las implicaciones que conllevan, se describen a continuación.

\subsubsection{Ausencia de los conocimientos sobre las mujeres y su experiencia}

El análisis de los datos extraídos de los documentos analizados revela que el 74,6\% en España y 97,1\% de las asignaturas en Brasil no se menciona ningún concepto relacionado con la perspectiva de género. Los datos indican una ausencia mayoritaria de enfoque acerca de las relaciones de género o referencias a las mujeres y su experiencia y realidad en las prácticas corporales.

En este nivel de discurso, cabe destacar de forma especial la ausencia del término "coeducación" en asignaturas obligatorias vinculadas a la pedagogía o a la didáctica. En Brasil, este término se encontró solo en dos documentos de instituciones distintas (B59 y B62), con apenas una citación cada una en el apartado de referencias bibliográficas. En el caso español, el concepto "coeducación" incrementa su frecuencia por el hecho de constar en una de las competencias del grado universitario de alguna universidad. Este es el caso de una universidad en el que redactan como competencia del título de grado "hábitos sociales y valores democráticos (coeducación de géneros, respecto a la diversidad social,..." (E9) por lo que se encuentra en la mayoría de GD de esta institución. En alguna otra ocasión, pero de forma escasa, aparece como temario a impartir: "Coeducación y equidad. Estrategias coeducativas en el desarrollo de los bloques de contenido de Educación Física" (E708), en una asignatura vinculada con la educación y con la transformación social denominada "Educación Física y diversidad: análisis y 
propuestas". De ello se desprendre que el enfoque coeducativo no parece ser trascendente en educación física, a pesar de que el marco normativo establece su necesidad y obligatoriedad (Serra, 2016).

También destaca la ausencia de la perspectiva de género en asignaturas vinculadas a la sociología o la historia de la actividad física y el deporte. A pesar que puede considerarse una categoría básica en los análisis socioculturales, son pocos los casos en los que se encuentran términos directamente relacionados con la perspectiva de género, por lo que se desprende que la categoría de género queda diluida bajo otras denominaciones genéricas como "aspectos socioculturales" o "derechos humanos" (Serra, 2016), lo que parece ser un dato común en ambos países investigados. Tal y como indica el estudio de Flintoff y Fitzgerald (2012), las cuestiones de género en la educación superior a menudo se consideran un tema periférico o secundario. Un ejemplo de ello es que el género acaba diluyéndose o fusionándose con otro tipo de diversidades que actualmente se consideran más relevantes, como puede ser la diversidad cultural o la educación adaptada (Prat y Flintoff, 2012; Serra et al., 2016b), lo cual contribuye a la invisibilización de esta temática. Esta situación puede ser comprendida desde una perspectiva androcéntrica del conocimiento, que viene siendo denunciada por Donoso-Vázquez, Montané y Pessoa (2014).

\subsubsection{Presencia superficial o 'políticamente correcta'}

En un $18,4 \%$ de las asignaturas españolas y en un 2,1\% de las asignaturas brasileras se constata la presencia de la perspectiva de género de un modo que puede ser considerada superficial o políticamente correcta, pero poco efectiva en la práctica. Las 205 asignaturas con este enfoque incluyen en algún punto términos vinculados a la perspectiva de género, aunque el término aparece en un solo apartado y no se encuentra otra alusión en otros puntos.

Los resultados están de acuerdo con la investigación realizada por Pezzi et al. (2011) que indican que la perspectiva de género está incluida en los apartados generales de los documentos institucionales o en las competencias generales del título, pero no se desarrollan y concretan en las asignaturas que conforman el proceso de formación docente, lo que caracteriza tan solo un discurso correcto y de acuerdo con lo esperado, pero que no asegura que se lleve realmente a cabo. 


\subsubsection{Reproduciendo a mujeres y a hombres}

En esta categoría se han identificado 27 (3,5\%) asignaturas en España y $6(0,1 \%)$ asignaturas en Brasil. Se trata de asignaturas que incluyen conocimientos relacionados con la mujer, si bien pueden conducir a reproducir los modelos tradicionales de género. En estos casos se observa que en ocasiones, si se aborda el conocimiento sobre mujeres, es para destacar su función reproductora, con temas como 'Actividad física durante el embarazo' (E360) o 'Actividades acuáticas para embarazadas y bebés' (E122), que son contenidos encontrados en asignaturas vinculadas a las actividades acuáticas, para poblaciones con necesidades específicas, fisiología del ejercicio, anatomía o entrenamiento deportivo. En algunos casos también se incluyen referencias a los aspectos biológicos y fisiológicos propios de las mujeres desde un enfoque patológico "Mujer y embarazo. Personas con determinadas patologías" (E453) o "Poblaciones especiales: menstruación y disfunción menstrual, embarazo, osteoporosis, desórdenes alimentarios" (E342). En esta cuestión, Goellner (2013) destaca que muchos discursos solo tienen en cuenta la feminidad que adopta la maternidad, de modo que se considera impropia cualquier actividad que ponga en peligro esta función.

Así mismo, en una institución del norte brasileño (B28), una asignatura vinculada a la temática de metodología del entrenamiento deportivo aborda las diferencias entre mujeres y hombres con términos que asocian a las mujeres a la fragilidad. En este sentido se constata como los estudios de educación física también estan impregnados de un discurso que fomenta el determinismo biológico, lo que termina por reproducir modelos de género hegemónicos que refuerzan la visión dicotómica y la relación de superoridad e inferioridad entre mujeres y hombres (Pfister, 2010).

La reproducción ocurre no solo en las asignaturas de cuña biológica, pero también en aquellas vinculadas a las ciencias humanas y sociales. Algunas, como la de " $\mathrm{Di}-$ mensões Sociológicas da Educação Física e do Esporte" (B50), fundamentándose en estadísticas de participación o la falta de interés de las niñas, acaban por generar un discurso acrítico donde las mujeres son el 'problema', pues participan menos y no están motivadas para la práctica deportiva, sin analizar que estas diferencias son construidas socialmente y a partir de relaciones de poder existentes en todas las interacciones sociales, en especial en las organizaciones deportivas donde impera una hegemonía del modelo androcéntrico. 


\subsubsection{Reconociendo lo femenino y las múltiples diferencias}

En esta categoría se identificaron a $20(2,6 \%)$ asignaturas en el caso español y $13(0,4 \%)$ asignaturas en el caso brasileño. Los elementos que caracterizan esta categoría tienen relación con el reconocimiento de las aportaciones de las mujeres y a sus experiencias con el deporte y la actividad física, buscando explicitar las formas de hacer de las mujeres por si mismas, así como las diferencias de las mujeres entre sí. Entre las asignaturas brasileñas identificadas en esta categoría se encuentra una asignatura de una universidad pública del sur del país con la denominación de "Dimensões do Processo Ensino-Aprendizagem da Educação Física I" (B59), en la que se pretende reflexionar sobre las experiencias de las chicas en educación física y su relación con la metodología de aprendizaje del movimiento, del deporte y de otras prácticas corporales. En ella se desprende la intención de desarrollar conceptos como inclusión, diferencia, o desigualdad, sexualidad y género, además de otras temáticas como clase social o etnia, como elementos esenciales para la formación profesional y el ejercicio docente. En esta línea, se presentan las diferencias y desigualdades entre mujeres y hombres considerando la importancia del proceso de socialización y las relaciones de poder en el marco del patriarcado.

Las asignaturas que discuten los significados culturales del cuerpo son importantes para el reconocimiento de las múltiples diferencias y propiciar rupturas con la visión dicotómica del ser humano. Es el caso de una asignatura que se encuentra en la malla curricular de una institución privada del nordeste brasileño, titulada "Fundamentos Antropológicos da Educação Física" (B23). Entre otros contenidos, aborda la temática de los usos y significados del cuerpo y las prácticas corporales y el deporte como expresión de la cultura y de la sociedad. Desde estas asignaturas se despliega el pensamiento de Marta Lamas que nos aporta la siguiente reflexión:

Si el cuerpo es el lugar donde la cultura aterriza los significados que le da la diferencia sexual, ¿cómo distinguir qué aspectos de ese cuerpo están libres de marca cultural, o sea, de género? No hay forma de responder a esta interrogante porque no hay cuerpo que no haya sido marcado por la cultura (Lamas, 1995, p. 56).

En el proceso de formación de los futuros y futuras profesoras, se considera relevante impartir una práctica docente desde la pedagogía crítica, en la que se reflexione sobre las desigualdades existentes entre hombres y mujeres, así como poner en evidencia los estereotipos de género. Es decir, es importante reflexionar sobre la influencia de las etiquetas sociales para elegir una actividad $u$ otra, ayudando a combatir el sesgo de género en aquellas actividades consideradas de hombres o de mujeres. Así mismo, es importante articular las cuestiones de género con las cuestiones de desigualdad racial y de clase social, desde una perspectiva interseccional, 
lo que potenciaría la lucha por los derechos humanos y la igualdad efectiva. Esta perspectiva se da en algunas GD de las asignaturas relacionadas con la sociología (E259) o la psicología (E510) argumentando que se debe comprender la relación entre la práctica de la actividad física en función del conjunto de características de las personas (edad, género, etnia y clase social).

\subsubsection{Trasformación de las relaciones de género}

De las 3074 asignaturas analizadas en Brasil, fue posible identificar que solo nueve $(0,2 \%)$ que tienen como eje central la transformación de las relaciones de género y el desarrollo de buenas prácticas para empoderar a las mujeres, rasgo que se encuentra en siete $(0,9 \%)$ de las 763 asignaturas españolas.

Por ejemplo, la asignatura de "Innovación y cambio sociocultural en la actividad física y el deporte" (E421) incopora propuestas para la transformación de los modelos y relaciones tradicionales de género, y experiencias que favorecen la participación de los diversos col-lectivos minoritarios. También la asignatura de "Sociologia de la Actividad Física y Deporte" (E9) se propone intervenir para modificar los modelos tradionales de género, y en la asignatura de "Planificación y organización de sistemas y actividades deportivas" (E119), se apuesta por desarrollar un plan de igualdad en un sistema deportivo. Así mismo, en la optativa "Mujer y deporte" (E280) se ofrece un enfoque holístico a la perspectiva de género considerando no sólo aquello que afecta a las mujeres, como puede desprenderse del título, sino que aporta una visión integral de la cuestión, incorporando también las distintas masculinidades.

De las nueve asignaturas brasileñas, apenas cuatro de ellas son obligatorias, mientras que las otras cinco son optativas, como la asignatura titulada "Gênero na Educação Física" (B62). En este contexto, hay instituciones que destacan, como por ejemplo una institución privada B21, ubicada en la región nordeste de Brasil. En esta institución hay una asignatura específica situada en el primer semestre del primer curso que tiene como título "Corpo, Gênero e Sexualidade", en la que se propone estudiar las construcciones histórico-sociales de género y sexualidad en la educación física en el contexto escolar. Se encuentra insertada en un conjunto de asignaturas que contribuyen a los conocimientos sociales y antropológicos de la educación física, con la intención de presentar cuestiones actuales del campo y necesarias al ejercicio de la docencia. Además de esta asignatura que es optativa, hay varias asignaturas obligatorias - "Ginástica, Esportes Individuais I e II, 
Esportes Coletivos I e II" (B21), impartidas en diferentes semestres a lo largo de los tres primeros años de la formación, que también incorporan la discusión de la perspectiva de género y se apoyan en bibliografía básica específica.

Sin embargo, en la visión de conjunto, estos casos son excepcionales. El análisis del global de los datos nos indica un panorama muy difícil en la formación docente desde la perspectiva de género en este campo, ya que lo poco que hay se presenta de forma puntual y con poca articulación con las otras asignaturas.

\section{Conclusiones}

Los efectos de la desigualdad de género en educación son múltiples y complejos, y están vinculados a factores estructurales y de contexto (Montané y Pessoa de Carvalho, 2012). Desde esta investigación, se ha podido observar, en primer lugar, que en los dos países existen marcos legales y administrativos por lo que se refiere a la inclusión de políticas de género en la educación superior, si bien con diferencias relevantes. En España, las leyes a favor de la igualdad y el marco de transformación del EEES han favorecido la incorporación de medidas para su inclusión en todos los estudios universitarios, ofreciendo un punto de partida favorable a la transformación de los modelos y relaciones tradicionales de género desde la educación superior. Por otra parte, en Brasil las normativas actuales y el contexto político no han propiciado un contexto favorecedor a su inclusión, sino más bien lo contrario, ya que se encuentra en una clara regresión (Nicolino et al., 2017; Nicolino, 2018), con la finalidad de distorsionar y eliminar los estudios de género del campo académico, por medio de la "dessubstancialização de seus sentidos histórico, socioantropológico e político" (Gonçalves y Mello, 2017). Al comparar los dos países, se destacan las diferencias en el desarrollo de políticas de igualdad: en Brasil, la desigualdad relacionada con el género convive con la disparidad de ingresos financeros y las diferencias de etnia y religión en un contexto cultural aún conservador y reaccionario. En España, a pesar de tener las políticas de igualdad más desarrolladas, los procesos de discriminación se convierten en más sutiles (Montané y Pessoa de Carvalho, 2012). En ambos casos se convive con diferentes formas de inequidad, además de violencias de género cotidianas.

A pesar de la diversidad de marcos políticos y sociales que presentan las dos realidades analizadas, por lo que se refiere a la presencia de la perspectiva de género en los currículos de formación docente en educación física, los resultados del estudio muestran situaciones similares. Coincidiendo con Tetreault (1985), los discursos 
pueden ayudar a la perpetuación de los roles de género y de la visión androcéntrica del mundo, o bien aportar conocimiento para ayudar en el cambio de la sociedad a través de la educación en cuestiones de género. A pesar de que los conocimientos sobre las relaciones de género se han extendido y multiplicado en los últimos años, se constata que en ambas realidades prevalece una mirada masculina en el campo de la educación física, la actividad física y los deportes, indicando un largo camino por delante hasta alcanzar la igualdad de género.

En ambos países la presencia de la perspectiva de género en la formación del futuro profesorado o profesional de la actividad física y el deporte es puntual y anecdótica, aunque se observa una presencia algo más fuerte en España, lo que tal vez pueda ser atribuido a su marco legal y al contexto de trasnformación social. Así, según se desprende de los resultados de este estudio, la poca concreción de las nuevas políticas, como denuncian Anguita y Torrego (2009), y la falta de propuestas claras para su introducción y puesta en práctica, llevan a que, finalmente, no se concreten en las guías docentes.

En Brasil, es posible afirmar que la presencia de la temática de género se encuentra de forma muy puntual y poco articulada con los objetivos planteados en la legislación para el proceso de formación docente en las instituciones. Se detecta su presencia en títulos y objetivos de algunas asignaturas, pero no se concreta en las referencias bibliográficas u otros apartados que subsidiarían la práctica docente. Poco se observa en los principios y objetivos generales de los proyectos pedagógicos y curriculares, prevaleciendo una perspectiva androcéntrica y sin hacer menciones más específicas a la igualdad de género $u$ otras cuestiones relacionadas a los derechos humanos.

Los resultados del estudio nos indican que en ambos contextos se hace necesaria una urgente revisión de las GD y PCC, y un compromiso institucional para que estas propuestas sean implementadas de forma efectiva, no unicamente para cumplir la normativa vigente, sino por el compromiso social que esto significa. La propuesta de categorizacion por niveles que se desprende de esta investigación puede servir de ayuda a los y las profesionales para reflexionar y situarse en alguna de las categorias sugeridas, y analizar que elementos puede incorporar o ampliar para que su docencia incorpore aspectos de mejora. De esta forma detectar si la perspectiva de género en sus asignaturas es ausente, superficial, reproductiva o transformadora.

Por otra parte, es necesario también aclarar las limitaciones de una investigación que se basa en documentos como evidencia empírica. Por su naturaleza, los documentos no muestran la versatilidad de un currículo "en acción" con la cultura local 
e institucional. No recogen, tampoco, las estrategias docentes durante el proceso de formación o los recursos didácticos (videos, mensajes, metodologías, reflexiones que utilizan en sus clases, etc.) que se ponen en práctica. Por el hecho de que los documentos incorporen términos vinculados a la perspectiva de género, no implica necesariamente que se introduzca en la docencia en el aula, y del mismo modo, que no se mencione, no implica que no se introduzca. Por ello, se propone como línea de trabajo futura analizar otros materiales curriculares del profesorado (como sus diapositivas, apuntes, etc.), así como su discurso en el aula.

A pesar de estas consideraciones, los datos nos indican un aspecto de la realidad que no es menor y no debería ser menospreciado o tratado como meramente administrativo. El estudio, en general, mostró que la discusión de género es puntual en el proceso de formación docente en educación física en ambos países, a pesar de los casi 70 años de la Declaración Universal de los Derechos Humanos y los más de 200 años de la Declaración de los Derechos de la Mujer y de la Ciudadana. Hemos avanzado, pero hay mucho por hacer en el campo de la formación docente y en el de la investigación con vistas a la equidad de género.

\section{Referencias bibliográficas}

Anguita, R., y Torrego, L. (2009). Género, educación y formación del profesorado. Retos y posibilidades. Revista Interuniversitaria de Formación del Profesorado, 64(23), 17-25.

Antas, R. C. y Arantes, A. S. (2019). Supressão do conceito de gênero em documentos de educação no Brasil: debates e contexto. Revista Cocar, 13(26), 393-407.

Blat, A. (1994). Informe sobre la igualdad de oportunidades educativas entre los sexos. Revista Iberoamericana de Educación, 6, 123-145. http://dx.doi.org/10.35362/rie601210

Bonder, G. (1994). Mujer y educación en América Latina: hacia la igualdad de oportunidades. Revista Iberoamericana de Educación, 6, 9-48. http://dx.doi. org/10.35362/rie601206

Brasil (1997). Parâmetros curriculares nacionais: introdução aos parâmetros curriculares nacionais. Brasília: Ministério da Educação e do Desporto/Secretaria de Educação Fundamental (MEC/SEF).

Chuppa-Cornell, K. (2005). The conditions of difficulty and struggle?: A discovered theme of curriculum transformation and women's studies discourse. NWSA Journal, 1(17), 23-24. http://dx.doi.org/10.2979/NWS.2005.17.1.23. 
Devís-Devís, J., Peiró, C., Molina, P. J., Villamón, M., Antolín, L., y Roda, F. (2001). Los materiales curriculares impresos en educación física: clasificación, usos e investigación. Movimento, 15(7), 119-136.

Donoso-Vázquez, T., Montané A. y Pessoa, M. E. (2014). Género y calidad en Educación Superior. Revista Electrónica Interuniversitaria de Formación del Profesorado, 17(3), 157-171. http://dx.doi.org/10.6018/reifop.17.3.204121

Flintoff, A. (1993). One of the boys? Gender identities in physical education initial teacher education. En I. Siraj-Blatchford (Ed.), Race, gender and the education of teachers (74-85). Buckingham: Open University Press.

Flintoff, A. y Fitzgerald, H. (2012). Theorizing Difference and in(Equality) in Physical Education, Youth Sport and Health. In F. Dowling, H. Fitzgerald y A. Flintoff (Eds.), Equity and Difference in Physical Education, Youth Sport and Health: A Narrative Approach (11-36). London: Routledge.

Goellner, S. (2013). A contribuição dos estudos de gênero e feministas para o campo acadêmico-profissional da Educação Física. En P. Dornelles (Ed.), Educação Física e gênero: desafios educacionais (23-44). Ijuí: Editora Unijuí.

Gonçalves, E., \& Mello, L. (2017). Apresentação: Gênero - Vicissitudes de Uma Categoria e Seus'Problemas.' Ciência e Cultura, 69(1), 26-30. https://doi.org/10.21800/2317$\underline{66602017000100012 .}$

Hernández, J., Gómez, R. H., Cinta, D., Carralero, A., y Altuve, E. (2008). Análisis de los contenidos curriculares de la formación de los docentes de Educación Física en Iberoamérica: los casos de Argentina, Chile, Costa Rica, Cuba, Ecuador, España, México, Paraguay, Perú y Venezuela. Revista Iberoamericana de Educación, 45(1), 1-16. https://doi.org/10.35362/rie4512166

Lamas, M. (1995). Usos, dificultades y posibilidades de la categoría género. Revista Estudios de Género, 1, 10-61.

Lledó, E. (2011). Manual de llengua per visibilitzar la presència femenina. Disponible en: http://www.beat.cat/manualdellengua/

Lei 9394/2006, de 20 de diciembre, que estabelece las diretrices y bases de educación nacional (DOU, de 23 de diciembre, 2016).

Ley Orgánica 1/1990, del 3 octubre, de Ordenación General del Sistema Educativo, BOE $238 \S 28927$ a 28942 (1990).

Ley Orgánica 3/2007, de 22 de marzo, para la igualdad efectiva de mujeres y hombres, BOE $71 \S 6115$ (2007).

Maher, F. A., y Tetreault, M. K. T. (2011). Long-term transformations: excavating privilege and diversity in the academy. Gender and Education, 23(3), 281-297. http:// dx.doi.org/10.1080/09540253.2010.491790.

McIntosh, P. (1984). Interactive phases of curricular re-vision: a feminist perspective. En B. Spanier, A. Bloom y D. Boroviak (Eds.), Toward a balanced curriculum: A 
sourcebook for initiating gender integrationrojects. Chicago: Schenkman Publishing Company.

Montané, A., y Pessoa de Carvalho, M. (2012). Diálogo sobre género: justicia, equidad y políticas de igualdad en educación superior (Brasil y España). Revista Lusófona de Educação, 21, 97-120.

Nicolino, A., Canon Buitrago, E., Campomar, G.C., Nicolás Patierno, M.G., Dogliotti, P., López de d'Amico, R. (2017). Educação Física e gênero: um estudo panorâmico da legislação e dos currículos de formação docente da América-Latina. En A. M Silva y V. Molina (Eds.), Educación Física en América Latina. Currículos y Horizontes Formativos (63-106). Anhangabaú-Jundiai: Paco Editorial.

Nicolino, A. (2018). Gênero nos currículos da formação docente em Educação Física no Brasil. En M. A. Paraíso y M. C. S Caldeira (Ed.), Pesquisas sobre currículos, gêneros e sexualidades (73-91). Belo Horizonte: Mazza.

ONU. (2015). Objetivos de desenvolvimento sustentável. Agenda 2030 para o Desenvolvimento Sustentável, Nova York.

ONU. (2019). Mulheres participa de sessão solene da Câmara por 13 anos da Lei Maria da Penha. Recuperado de https://bit.ly/2MzvbRE

Pereira e Silva, I., Pereira da Silva, J. M., Grabowski, G., \& Zucchetti, D. T. (2017). As competências para o século XXI na perspetiva dos professores Um estudo na região centro-oeste de Portugal. Revista Iberoamericana de Educación, 74, 193-216. https://doi.org/10.35362/rie740614

Pezzi, P., López, I., Marchant, A., Méndez, M. T., Ramos, E. M., Ruiz, M. B., y Ybáñez, P. (2011). Universidad y feminismo: la integración transversal de los estudios de género en las prácticas docentes en Humanidades. Comunicación presentada en Congreso Internacional Educación para la igualdad: Género y Sexualidades, Granada.

Pfister, G. (2010). Women in sport-gender relations and future perspectives. Sport in Society, 13(2), 234-248. http://dx.doi.org/10.1080/17430430903522954.

Plano Nacional de Educação, que define o plano nacional de educação para a década (2014-2024), Ministério da Educação/Brasil.

Prat, M., y Flintoff, A. (2012). Tomando el pulso a la perspectiva de género: un estudio de caso en la formación del profesorado de Educación Física. Revista Electrónica Interuniversitaria de Formación del Profesorado, 15(3), 69-83.

Resolução Conselho Nacional de Educação, de 1 de julio de 2015, CNE Nº 02 de 2015, Ministério da Educação/Brasil (2015).

Rosado-Nunes, M. J. F. (2015). A "ideologia de gênero" na discussão do PNE. A intervenção da hierarquia católica. HORIZONTE - Revista De Estudos De Teologia E Ciências Da Religião, 13(39), 1237-1260. http://dx.doi.org/10.5752/P.2175$\underline{5841.2015 v 13 n 39 p 1237}$ 
Scraton, S. (2013). Feminism and Physical Education: does gender still matter?. En G. Pfister yM.K. Sisjord coord). Gender and Sport, Changes and challenges. Münster. Vaxmann Verlag GmbH, 199-216.

Seibold, J. R. (2000). La calidad integral en educación. Reflexiones sobre un nuevo concepto de calidad educativa que integre valores y equidad educativa. Revista Iberoamericana de Educación, 23, 215-231. http://dx.doi.org/10.35362/rie2301012

Serra, P. (2016). La perspectiva de género en los estudios de ciencias de la actividad física y el deporte. Tesis doctoral - Univeristat de Barcelona. http://hdl.handle. net/10803/402513

Serra, P., Vizcarra, M.T., Garay, B., Prat, M., y Soler, S. (2016a). Análisis del discurso de género en las matrices curriculares de las ciencias de actividad física y el deporte. Movimento, Porto Alegre, 22 (3), 821-834. http://dx.doi.org/10.22456/1982-8918.56959

Serra, P., Soler, S., Prat, M., Bizkarra, M., Garay, B., y Flintoff, A.(2016b). The (in)visibility of gender knowledge in the Physical Activity and Sport Science Degree in Spain. Sport, Education and Society. http://dx.doi.org/10.1080/13573322.2016.1199016.

Svendsen, A. M., y Svendsen, J. T. (2016). Teacher or coach? How logics from the field of sports contribute to the construction of knowledge in physical education teacher education pedagogical discourse through educational texts. Sport, Education and Society, 21(5), 796-810. http://dx.doi.org/10.1080/13573322.2014.956713.

Tetreault, M. K. (1985). Feminist Phase Theory: An Experience-Derived Evaluation Model. The Journal of Higher Education, 56(4), 363-384. http://dx.doi.org/10.2307/1981301.

UNESCO (2013). Relatório Final do Seminário Educação em sexualidade e relações de gênero na formação inicial docente no ensino superior. São Paulo: Fundação Carlos Chagas.

Weiner, G. (2000). A critical review of gender and teacher education in Europe. Pedagogy, Culture y Society, 8(2), 233-247. http://dx.doi.org/10.1080/14681360000200091. 